\title{
Molecular evolution and functional divergence of the bestrophin
} protein family

\author{
Vladimir M Milenkovic ${ }^{1,2}$, Thomas Langmann¹, Rainer Schreiber², \\ Karl Kunzelmann² and Bernhard HF Weber*1
}

\author{
Address: ${ }^{1}$ Institute of Human Genetics, University of Regensburg, Regensburg, Germany and 2Institute of Physiology, University of Regensburg, \\ Regensburg, Germany \\ Email: Vladimir M Milenkovic - milenkovic@eye-regensburg.de; Thomas Langmann - thomas.langmann@klinik.uni-regensburg.de; \\ Rainer Schreiber - rainer.schreiber@vkl.uni-regensburg.de; Karl Kunzelmann - karl.kunzelmann@vkl.uni-regensburg.de; \\ Bernhard HF Weber* - bweb@klinik.uni-regensburg.de \\ * Corresponding author
}

Published: 28 February 2008

BMC Evolutionary Biology 2008, 8:72 doi:10.1 |86/|47|-2/48-8-72

This article is available from: http://www.biomedcentral.com/I47/-2/48/8/72

(c) 2008 Milenkovic et al; licensee BioMed Central Ltd.

This is an Open Access article distributed under the terms of the Creative Commons Attribution License (http://creativecommons.org/licenses/by/2.0), which permits unrestricted use, distribution, and reproduction in any medium, provided the original work is properly cited.
Received: 18 September 2007

Accepted: 28 February 2008

\begin{abstract}
Background: Mutations in human bestrophin I are associated with at least three autosomaldominant macular dystrophies including Best disease, adult onset vitelliform macular dystrophy and autosomal dominant vitreo-retinochoroidopathy. The protein is integral to the membrane and is likely involved in $\mathrm{Ca}^{2+}$-dependent transport of chloride ions across cellular membranes. Bestrophin I together with its three homologues forms a phylogenetically highly conserved family of proteins.
\end{abstract}

Results: A bioinformatics study was performed to investigate the phylogenetic relationship among the bestrophin family members and to statistically evaluate sequence conservation and functional divergence. Phylogenetic tree assembly with all available eukaryotic bestrophin sequences suggests gene duplication events in the lineage leading to the vertebrates. A common $\mathrm{N}$-terminal topology which includes four highly conserved transmembrane domains is shared by the members of the four paralogous groups of vertebrate bestrophins and has been constrained by purifying selection. Pairwise comparison shows that altered functional constraints have occurred at specific amino acid positions after phylogenetic diversification of the paralogues. Most notably, significant functional divergence was found between bestrophin 4 and the other family members, as well as between bestrophin 2 and bestrophin 3. Site-specific profiles were established by posterior probability analysis revealing significantly divergent clusters mainly in two hydrophilic loops and a region immediately adjacent to the last predicted transmembrane domain. Strikingly, codons 279 and 347 of human bestrophin 4 reveal high divergence when compared to the paralogous positions strongly indicating the functional importance of these residues for the bestrophin 4 protein. None of the functionally divergent amino acids were found to reside within obvious sequences patterns or motifs.

Conclusion: Our study highlights the molecular evolution of the bestrophin family of transmembrane proteins and indicates amino acid residues likely relevant for distinct functional properties of the paralogues. These findings may provide a starting point for further experimental verifications. 


\section{Background}

The bestrophins are a phylogenetically conserved family of integral membrane proteins initially identified in Caenorhabditis elegans [1]. Homologous sequences are found in animals, fungi, and prokaryotes, but not in protozoans or plants [2]. Conservation is mainly restricted to the N-terminal 350-400 amino acids with an invariant motif arginine-phenylalanine-proline (RFP) of unknown functional properties.

The first human bestrophin cloned, bestrophin 1, is encoded by the vitelliforme macular dystrophy type 2 (VMD2) gene on chromosome $11 \mathrm{q} 13$ and was shown to be associated with Best macular dystrophy (BMD, OMIM \#153700) also known as Best disease [3,4]. Subsequently, mutations in this gene were also found to cause adult onset vitelliform macular dystrophy (AVMD, OMIM \#608161) and autosomal dominant vitreo-retinochoroidopathy (ADVIRC, OMIM \#193220). The currently known 106 disease-causing mutations [5] are associated with a dominant pattern of inheritance with the majority being missense mutations located in four clusters near the RFP motif and the predicted transmembrane domains (TMDs) [6].

Based on immunocytochemical studies in macaque, porcine and human eyes, bestrophin 1 was shown to localize to the basolateral plasma membrane of the retinal pigment epithelium (RPE) $[7,8]$. In addition, bestrophin 1 is broadly expressed in other epithelia including the intestine and the lung [9], whereas bestrophin 2 expression appears confined to the olfactory epithelium [10]. On the functional level, whole cell patch clamp experiments suggest that the bestrophins act as $\mathrm{Ca}^{2+}$-dependent transporters of chloride ions across epithelial borders [11-16]. Functional proteins likely exist as multimeric complexes eventually by forming homo- or heteromeric associations between bestrophin family members.

Uncertainty exists as to the specific function of bestrophin 1. As an alternative to its activity as a chloride channel, bestrophin 1 may act as an accessory protein in the regulation of voltage-gated calcium channels [17]. In addition, bestrophin 1 could be involved in the volume sensitivity of RPE cells during phagocytosis of photoreceptor outer segments [11]. Consequently, distinct functional aspects of bestrophin 1 may contribute to RPE dysfunction and thus may explain the variable phenotypes associated with a mutant protein.

Phylogenetic studies of protein families can be a valuable tool to determine conserved but also divergent regions, potentially leading to functional predictions [18]. In this study we elucidated the evolutionary history of the bestrophins and identified structural and putative func- tional motifs of the bestrophin protein family by a comprehensive bioinformatics/phylogenetic approach. This has led us to predict distinct amino acid residues that may be of importance in the functional divergence of the bestrophin paralogues.

\section{Results \\ Phylogenetic analysis of the bestrophin family}

We first retrieved the available bestrophin sequences from the currently sequenced genomes. Querying major databases and unfinished genomes with the full-length amino acid sequences from the four human bestrophin paralogues identified 173 homologous proteins in vertebrates (mammals, birds, amphibians and fishes), urochordates (sea squirt), and invertebrates (insects, nematodes) (see Additional file 1). While C. elegans reveals a large number of bestrophins $(n=26)$, most other organisms harbour three or four family members. In each of the recently sequenced genomes of the urochordata Ciona inestinalis and Ciona savigny, the closest relatives of the craniates, we identified only a single bestrophin sequence strongly suggesting that gene duplication events may have occurred in the lineage leading to the vertebrates.

Our further study focused on vertebrate bestrophins. After exclusion of unfinished and partial protein sequences, a phylogenetic tree with 53 selected bestrophins (Table 1) was constructed by the neighbour-joining (NJ) method based on a gamma corrected Jones-Taylor-Thornton (JTT) distance matrix $[19,20]$ (Figure 1). Accordingly, the vertebrate bestrophins can unambiguously be separated into distinct clusters. With the urochordata as outgroup, the vertebrate bestrophins form four separate monophyletic groups, all with high bootstrap support indicating that the formation of the paralogous subfamilies occurred before the divergence of individual species (Figure 1). The phylogenetic branches of bestrophins 1 and 3 separated considerably earlier in evolution than bestrophin subgroups 2 and 4, partially explaining the level of sequence conservation between each of the two subfamilies. The high level of sequence identity within a subfamily suggests evolutionarily conserved functions. Overall, the data indicate that the bestrophin subfamilies have evolved by gene duplication events, in good agreement with previous findings demonstrating that large-scale gene duplications have occurred during chordate evolution [21,22].

\section{$\mathbf{N}$-terminal topology of the vertebrate bestrophins}

We next analyzed whether the strong sequence conservation between the $\mathrm{N}$-terminal regions of the vertebrate bestrophins also predicts a common topology. Putative transmembrane domains and hydrophobic regions were modelled in silico (Figure 2). Hydropathy plotting of 53 bestrophins (orthologues and paralogues, Table 1) revealed six hydrophobic regions including four putative 
Table I: Vertebrate bestrophin homologues $(n=53)$ used for phylogenetic analysis

\begin{tabular}{|c|c|c|c|c|c|c|c|}
\hline No. & $\begin{array}{l}\text { Protein } \\
\text { Name }\end{array}$ & $\begin{array}{l}\text { Short } \\
\text { name }\end{array}$ & Organism & Taxonomy & Amino acids & Gene bank identifier & cDNA Acc. No. \\
\hline 1 & Bestrophin I & $\mathrm{HsBI}$ & Homo sapiens & Mammalia & 585 & NP 004174 & AF07350I \\
\hline 2 & Bestrophin 2 & $\mathrm{HsB} 2$ & & & 509 & AAR99655 & AY5I5705 \\
\hline 3 & Bestrophin 3 & $\mathrm{HsB} 3$ & & & 668 & $\overline{N P \quad 1 / 6124}$ & NM_032735 \\
\hline 4 & Bestrophin 4 & $\mathrm{HsB} 4$ & & & 473 & NP 695006 & NM_I53274 \\
\hline 5 & Bestrophin I & PtBI & Pan troglodytes & Mammalia & 585 & $\underline{X P 001151529}$ & XM_00II5I529 \\
\hline 6 & Bestrophin 2 & PtB2 & & & 509 & XP 512414 & XM_5I24I4 \\
\hline 7 & Bestrophin 3 & PtB3 & & & 668 & $\overline{X P 522466}$ & XM_522466 \\
\hline 8 & Bestrophin 4 & PtB4 & & & 473 & $\overline{X P 524571}$ & XM_52457I \\
\hline 9 & Bestrophin I & $\mathrm{MmBI}$ & Macaca mulatta & Mammalia & 585 & $\mathrm{XP001116583}$ & XM_00III 6583 \\
\hline 10 & Bestrophin 2 & $\mathrm{MmB2}$ & & & 509 & $\mathrm{XP} 001108800$ & XM_00II 08800 \\
\hline 11 & Bestrophin 3 & $\mathrm{MmB3}$ & & & 669 & XP 001117392 & XM_00IIII7392 \\
\hline 12 & Bestrophin 4 & MmB4 & & & 473 & $\overline{X P 001098771}$ & XM_00109877I \\
\hline 13 & Bestrophin I & MfBI & Macaca fascicularis & Mammalia & 585 & AAQ56049 & AY357925 \\
\hline 14 & Bestrophin I & $\mathrm{BtBI}$ & Bos taurus & Mammalia & 589 & XP 585778 & XM_585778 \\
\hline 15 & Bestrophin 2 & $\mathrm{BtB2}$ & & & 528 & XP 607911 & XM_60791I \\
\hline 16 & Bestrophin 3 & $\mathrm{BtB3}$ & & & 369 & $\overline{X P \quad 613863}$ & $X M \_613863$ \\
\hline 17 & Bestrophin 4 & BtB4 & & & 467 & $\overline{X P 587691}$ & XM_58769I \\
\hline 18 & Bestrophin 3 & LaB3 & $\begin{array}{l}\text { Loxodonta } \\
\text { africana }\end{array}$ & Mammalia & 674 & ENSLAFP00000000778 & ENSLAFT00000000928 \\
\hline 19 & Bestrophin 2 & $\mathrm{FcB} 2$ & Felis catus & Mammalia & 488 & ENSFCAP00000000436 & ENSFCAT00000000470 \\
\hline 20 & Bestrophin I & $\mathrm{CfBI}$ & Canis familiaris & Mammalia & 693 & XP 540912 & XM_540912 \\
\hline 21 & Bestrophin 2 & $\mathrm{CfB2}$ & & & 509 & XP 542045 & XM_542045 \\
\hline 22 & Bestrophin 4 & CfB4 & & & 437 & XP 539638 & XM_539638 \\
\hline 23 & Bestrophin I & $\mathrm{MsBI}$ & Mus musculus & Mammalia & 551 & NP 036043 & NM_011913 \\
\hline 24 & Bestrophin 2 & MsB2 & & & 508 & AAS09923 & AY450428 \\
\hline 25 & Bestrophin 3 & MsB3 & & & 669 & NP 001007584 & NM_00I007583 \\
\hline 26 & Bestrophin I & $\mathrm{RnBI}$ & Rattus norvegicus & Mammalia & 550 & NP_00I0II940 & NM_00I0II 940 \\
\hline 27 & Bestrophin 2 & $\mathrm{RnB2}$ & & & 508 & $\underline{X P 001070841}$ & XM_00107084I \\
\hline 28 & Bestrophin 3 & $\mathrm{RnB3}$ & & & 672 & XP 235161 & XM_235I6I \\
\hline 29 & Bestrophin 4 & $\mathrm{RnB} 4$ & & & 454 & XP 001066317 & XM_001066317 \\
\hline 30 & Bestrophin I & MdBI & $\begin{array}{c}\text { Monodelphis } \\
\text { domestica }\end{array}$ & Mammalia & 467 & $\mathrm{XP} \mathrm{001363751}$ & XM_00I3637I4 \\
\hline 31 & Bestrophin 3 & MdB3 & & & 676 & XP 001369557 & XM_00I369520 \\
\hline 32 & Bestrophin 4 & MdB4 & & & 487 & $\underline{X P 001376079}$ & XM_001376042 \\
\hline 33 & Bestrophin 4 & OgB4 & Otolemur garnettii & Mammalia & 471 & ENSOGAP00000006199 & ENSOGAT00000006927 \\
\hline 34 & Bestrophin 4 & MIB4 & Myotis lucifugus & Mammalia & 473 & ENSMLUP0000000I 435 & ENSMLUT0000000I564 \\
\hline 35 & Bestrophin 3 & $\mathrm{OaB3}$ & $\begin{array}{l}\text { Ornithorhynchus } \\
\text { anatinus }\end{array}$ & & 675 & ENSOANP00000009484 & ENSOANT00000009486 \\
\hline 36 & Bestrophin I & $\mathrm{GgBI}$ & Gallus gallus & Aves & 762 & XP 421055 & XM_421055 \\
\hline 37 & Bestrophin 3 & GgB3 & & & 669 & $\overline{X P 416091}$ & XM_4I609I \\
\hline 38 & Bestrophin 4 & GgB4 & & & 488 & $\times 001234941$ & XM_00I234940 \\
\hline 39 & Bestrophin I & Xt-I & Xenopus tropicalis & Amphibia & 419 & ENSXETP000000I4724 & ENSXETTO00000। 4724 \\
\hline 40 & Bestrophin 2 & $x_{t-2}$ & & & 510 & NP 988974 & NM_203643 \\
\hline 41 & Bestrophin 3 & $X_{t-3}$ & & & 366 & ENSXETP00000002984 & ENSXETT00000002984 \\
\hline 42 & Bestrophin 4 & $\mathrm{xt}-4$ & & & 367 & ENSXETP0000002979I & ENSXETT0000002979। \\
\hline 43 & Bestrophin I & Tr-I & Takifugu rubripes & Actinopterygii & 369 & SINFRUP00000I4I703 & SINFRUT00000I4I703 \\
\hline 44 & Bestrophin 2 & $\operatorname{Tr}-2$ & & & 431 & SINFRUP00000I5II 23 & SINFRUT00000I5II 23 \\
\hline 45 & Bestrophin 3 & Tr-3 & & & 407 & SINFRUP00000I8I928 & SINFRUT00000I82495 \\
\hline 46 & Bestrophin 4 & $\operatorname{Tr}-4$ & & & 370 & SINFRUP00000I 34584 & SINFRUT00000I 34584 \\
\hline 47 & Bestrophin I & DrBI & Danio rerio & Actinopterygii & 367 & XP 689098 & XM_684006 \\
\hline 48 & Bestrophin 2 & DrB2 & & & 589 & $\overline{X P \quad 695597}$ & XM_690505 \\
\hline 49 & Bestrophin I & Tn-I & $\begin{array}{l}\text { Tetraodon } \\
\text { igrovoridis }\end{array}$ & Actinopterygii & 572 & CAG08784 & CAAEOIOI 5000 \\
\hline 50 & Bestrophin 2 & $\mathrm{Tn}-2$ & & & 431 & CAG03298 & CAAE010147| 2 \\
\hline 51 & Bestrophin 2 & Ga-2 & $\begin{array}{l}\text { Gasterosteus } \\
\text { culeatus }\end{array}$ & Actinopterygii & 368 & ENSGACP00000025248 & ENSGACT00000025297 \\
\hline 52 & Bestrophin 3 & Ga-3 & & & 367 & ENSGACP000000I 9354 & ENSGACT000000I9392 \\
\hline 53 & Bestrophin I & Ol-I & Oryzias latipes & Actinopterygii & 454 & ENSORLP000000077। 4 & ENSORLT000000077I 5 \\
\hline
\end{tabular}




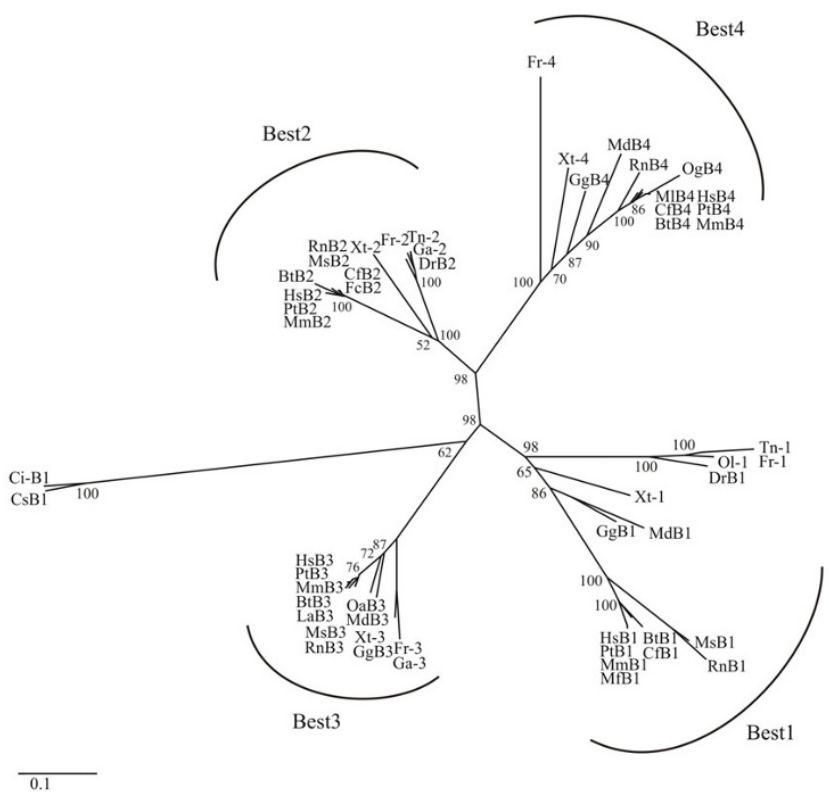

Figure I

Phylogenetic tree of $\mathbf{5 3}$ homologues of vertebrate bestrophins. The phylogenetic tree of the bestrophin protein family was inferred by the neighbor-joining method (NJ) method applying gamma corrected distances. Urochordata (Ciona intestinalis [Ci] and Ciona savigny [Cs]) were used as out-group. The support for each phylogenetic group was tested with 1000 bootstrap pseudoreplicates. Protein sequences are given in Table I.

transmembrane domains with hydropathy values reaching or above the threshold of 1.6 (Figure 2). These four hydrophobic peaks are strongly conserved in the vertebrate bestrophins suggesting membrane insertion of all bestrophin family members similar to bestrophin 1 [23].

\section{Variable selective pressures among amino acid sites}

To analyze positive or negative selection of specific amino acid regions within the full-length protein sequences of mammalian bestrophins, substitution rate ratios of nonsynonymous $(\mathrm{dN})$ versus synonymous (dS) mutations $(\mathrm{dN} / \mathrm{dS}$ or $\omega)$ were calculated for vertebrate bestrophins as given in Table 1 . A dN/dS value $<1$ is indicative of purifying selection acting against amino acid changes, whereas $\mathrm{dN} / \mathrm{dS}$ values $>1$ indicate an excess of amino acid changes suggesting adaptive evolution $[24,25]$. Amino acids in a protein sequence are expected to be under different selective pressure and to have different underlying $\mathrm{dN} / \mathrm{dS}$ ratios. Table 2 shows that the ratio values for the vertebrate bestrophins are substantially lower than 1 suggesting that the $\mathrm{N}$-termini of mammalian bestrophins within each subgroup were under strong purifying selection pressure. In order to test for positive selection at individual amino acid codons, the site-specific models implemented in codeml were used. Likelihood rate tests (LRTs) were

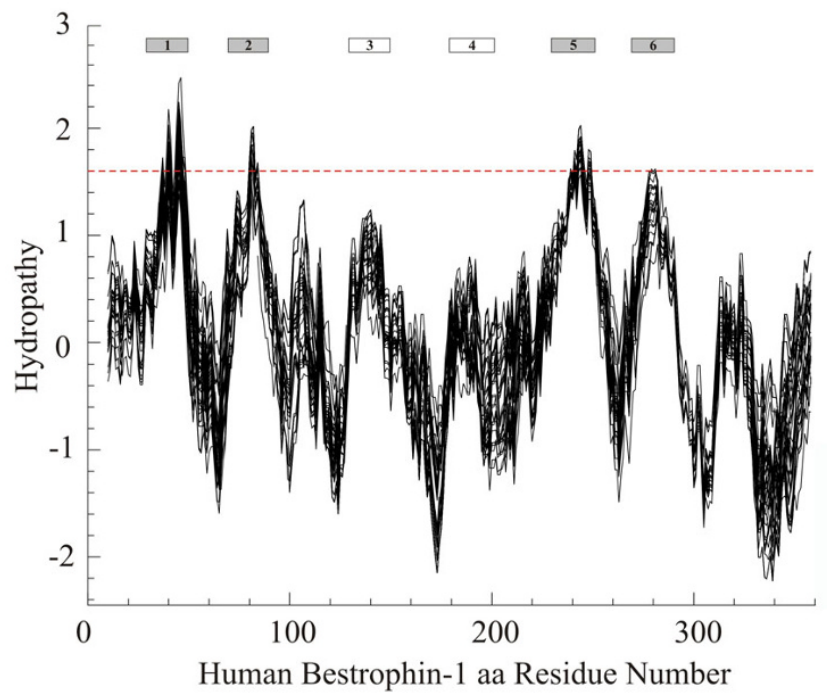

Figure 2

Average hydropathy plot of 53 homologues of vertebrate bestrophins. Hydropathy plot was generated from 53 protein sequences as given in Table I. A threshold value of $\mathrm{I} .6$ is marked by the dotted line. Positive values indicate hydrophobic regions. Putative transmembrane domains were determined by the TOPPRED II algorithm and are depicted as gray and white boxes on the top.

performed between model M0 (one ratio) and M3 (discrete), M1a (nearly neutral) and M2a (positive selection), and M7 (beta) and M8 (beta and $\omega$ ) (Table 2). The selection model (M2a) does not suggest presence of positively selected sites $(\mathrm{P}=1)$. M0 was rejected when compared to M3 $(\mathrm{P}<0.001)$, although no positively selected sites were detected. This could be explained by the fact that the majority of the protein is subjected to constant purifying selection, while a few sites undergo positive selection [26]. From the three models which allow for selection to be tested (M2a, M3 and M8), only M8 was significantly favoured over M7 $(P<0.001)$ and detected 44 sites at the 95\% level (Table 2). This provides evidence for the Darwinian selection in vertebrate bestrophins.

\section{Analysis of functional divergence}

To further investigate whether amino acid substitutions in the highly conserved N-termini of the bestrophins could have caused adaptive functional diversification, amino acid residues 1-367 from 53 vertebrate bestrophins (Table 1) were used for posterior analysis by the DIVERGE program algorithms $[27,28]$. Pairwise comparisons of paralogous bestrophins from subfamilies 1 to 4 were carried out and the rate of amino acid evolution at each sequence position was estimated. This identified residues potentially responsible for functional divergence. The results are given as a coefficient of functional divergence $(\theta)$ with standard errors and significance levels (Table 3). Signifi- 
Table 2: Likelihood values and parameter estimates for the vertebrate bestrophin genes

\begin{tabular}{|c|c|c|c|c|}
\hline Model & $\mathbf{d N} / \mathbf{d S}^{\mathrm{a}}$ & Estimates of parameters ${ }^{b}$ & $\mathbf{I}$ & Positively Selected Sites ${ }^{c}$ \\
\hline MO (one-ratio) & 0.053 & $\omega=0.053$ & -22547.97 & not allowed \\
\hline MIa (nearly neutral) & 0.084 & $\begin{array}{l}P_{0}=0.964,\left(P_{1}=0.036\right) \\
\left(\omega_{0}=0.050\right),\left(\omega_{1}=1\right)\end{array}$ & -22443.90 & not allowed \\
\hline M2a (positive selection) & 0.084 & $\begin{array}{l}P_{0}=0.964, P_{1}=0.002,\left(P_{2}=0.033\right) \\
\left(\omega_{0}=0.050\right),\left(\omega_{1}=1\right), \omega_{2}=1\end{array}$ & -22443.90 & not found \\
\hline M3 (discrete) & 0.058 & $\begin{array}{l}P_{0}=0.529, P_{1}=0.355,\left(P_{2}=0.115\right) \\
\omega_{0}=0.01 \mathrm{I}, \omega_{1}=0.073, \omega_{2}=0.230\end{array}$ & -22016.13 & not found \\
\hline M7 (beta) & 0.063 & $p=0.598, q=8.411$ & -22007.24 & not allowed \\
\hline M8 (beta\& $\omega)$ & 0.220 & $\begin{array}{l}P_{0}=1,\left(P_{1}=0\right) \\
P=0.685, q=1.988, \omega=4.997\end{array}$ & -19662.03 & $\begin{array}{l}8,25,40,41,42,44,45^{*}, 48,49,52^{*}, 53,55,56,57,60^{*}, \\
61 *, 67,7 I, 95,108,113,117,120, I 54,159,165^{*}, 170,173, \\
175,193,197,207^{*}, 209^{*}, 216,219,26 I, 263^{*}, 265^{*}, 33 I^{*}, \\
340,34 I, 342,344,354^{*}\end{array}$ \\
\hline
\end{tabular}

a The $\mathrm{dN} / \mathrm{dS}$ ratio is an average over all sites of bestrophin gene alignments.

b Parameters in parentheses are not free parameters.

${ }^{c}$ Numbering of amino acid residues corresponds to human bestrophin I. Positive selection sites with posterior probabilities $>95 \%$ are shown in bold.

* The amino acid residues depicted with an asterisk were also found to be implicated in the functional divergence between bestrophin paralogues (see Table 4).

Table 3: Functional divergence estimated in 53 vertebrate bestrophin paralogues

\begin{tabular}{llllll}
\hline Comparison & $\theta^{\mathrm{a}}$ & $\mathbf{S E}^{\mathrm{b}}(\theta)$ & LRT $^{\mathrm{c}}(\theta)$ & $\mathbf{P}^{\mathrm{d}}$ & Probability cutoffe \\
\hline BestI vs Best2 & 0.097 & 0.076 & 1.640 & 0.024541 & - \\
Best I vs Best3 & 0.142 & 0.043 & 10.762 & 0.000488 & - \\
Best I vs Best4 & 0.217 & 0.039 & 30.099 & $8.88557 \mathrm{e}-8$ & 0.70 \\
Best2 vs Best3 & 0.380 & 0.073 & 27.152 & $6.32074 \mathrm{e}-9$ & 0.80 \\
Best2 vs Best4 & 0.320 & 0.075 & 18.120 & $9.855 \mathrm{e}-10$ & 0.64 \\
Best3 vs Best4 & 0.260 & 0.059 & 19.338 & $9.477 \mathrm{e}-10$ & 0.70 \\
\hline
\end{tabular}

a $\theta$ is the coefficient of functional divergence.

b SE, standard error.

c LRT $(\theta)$ is a likelihood ratio test.

${ }^{d}$ significance level $\left(\mathrm{Pc}^{\mathrm{C}}\right)$ calculated by the method of Fisher's transformation and corrected by Bonferroni.

e Probability cut-off is the minimal posterior probaility for amino acids causing functional divergence.

cant functional divergence was detected between bestrophin 2 and $3\left(\mathrm{P}_{\text {corr }}=6.3 \times 10^{-9}\right)$ as well as between bestrophin 4 and the other bestrophin subgroups $\left(\mathrm{P}_{\text {corr }} \leq\right.$ $\left.8.9 \times 10^{-8}\right)$.

Next, single amino acid residues responsible for the functional divergence were predicted based on site-specific profiles (Figure 3A) in combination with suitable cut-offvalues derived from the posterior probability of each comparison (Table 3). Residues predicted to be functionally divergent in bestrophin, were mapped onto topology models of human bestrophins 2 and 4, respectively (Figure $3 \mathrm{~b}$ ). The predicted functional sites are not equally distributed throughout the respective bestrophin, but instead are clustered in the hydrophilic loops between predicted transmembrane domains 1 and 2 as well as 5 and 6 and immediately C-terminal to transmembrane domain 6 (Figure 3B, see Additional file 2, and Table 4). Despite the high global sequence identity of mammalian bestrophins
2 and 3, functionally divergent amino acids were also identified between these bestrophins. For example, at position 264 (numbered according to bestrophin 1) the tyrosine residue is completely conserved within bestrophin 1, 2 and 3 paralogues but highly divergent in bestrophin 4 paralogues. Similarly, residue 331 (numbered according to bestrophin 1) is highly divergent in bestrophin 1, 2 and 3 paralogues but strictly conserved in bestrophins 4 (Figure 3B, see Additional file 2, and Table 4). We thus speculate that these two sites may be of functional importance for the respective bestrophin subfamilies. In addition, 11 out of 44 positively selected sites detected by LRT in codeml were also found to be functionally divergent between bestrophin paralogues (marked by asterisk in Table 4).

We finally scanned the predicted divergent amino acids and surrounding sequences in bestrophins 2 and 4 for the presence of domains and functional sequence patterns 
A
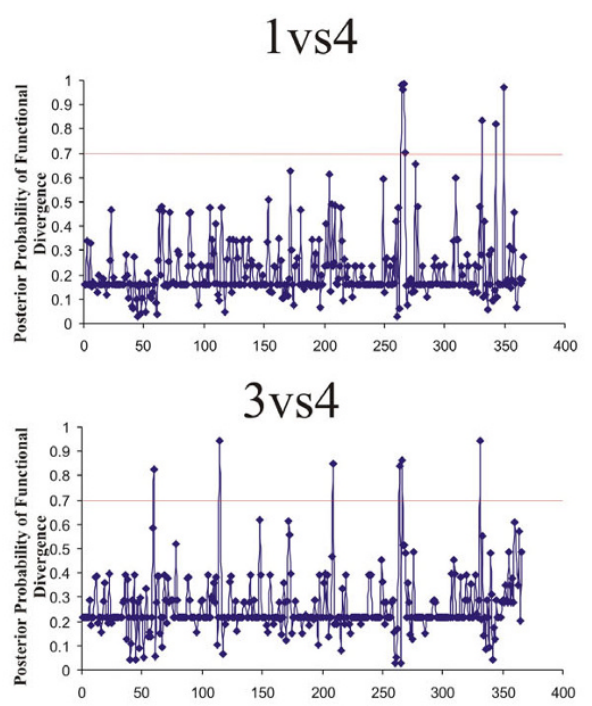
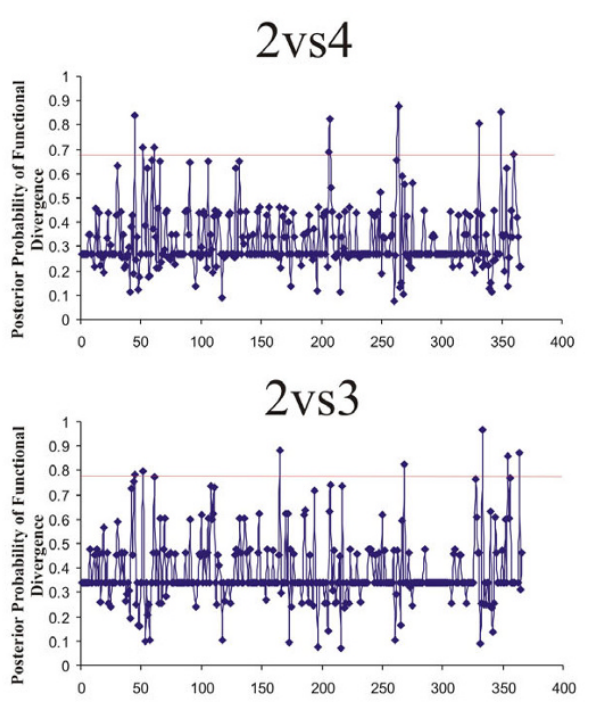

B
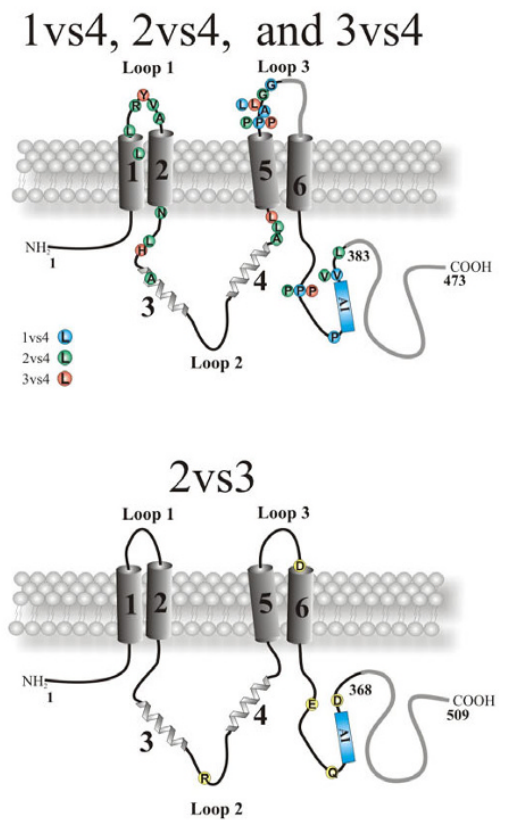

Figure 3

Site specific profiles for evolutionary rate changes in the vertebrate bestrophin protein family. A, The posterior probabilities of functional divergence for vertebrate bestrophins I to 4 were obtained with Diverge [27]. Individual cut-off values for each comparison are marked with red horizontal lines. B, Residues with predicted functional divergence between bestrophin subfamilies are mapped onto the membrane topology models of bestrophin 4 (top) and bestrophin 2 (bottom). Divergent amino acids are shown and listed in Table 4. In the model drawing, residues excluded from the analysis are depicted in gray.

with MotifScan and InterProScan. Identity to known protein motifs, except for several short sequence tags including putative phosphorylation sites, could not be detected.

\section{Discussion}

Our phylogenetic analysis suggests that the bestrophins originated by duplication and divergence of a common protein at the base of the eukaryotic tree, some $700 \mathrm{Myr}$ ago. Analysis of the bestrophins from a phylogenetic perspective may provide the basis for understanding the functional diversity within this conserved protein family. Both small-scale and large-scale gene duplications are known to contribute to the complexity of eukaryotic organisms $[29,30]$. The presence of bestrophins in prokaryotes [2] and their occurrence in phylogenetically distant eukaryotic species such as insects and mammals highlight their general functional importance.

High levels of sequence homology were generally found between the N-terminal regions of the bestrophins, while the C-termini differ substantially particularly between paralogues. At the N-terminal sequences, vertebrate bestrophins show nearly identical hydrophobicity plots with four major and two minor hydrophobic peaks, indicating that they share a highly similar membrane topology $[16,23]$. The hydrophobic regions with high sequence conservation also seem to contribute to oligomerization $[15,31]$ and the second putative transmembrane domain is thought to be especially important for channel function $[13,14,32]$.

Our data and the study by Hagen et al. [2] demonstrate that the vertebrate bestrophins cluster in organismal groups, i.e. the homologues are more related to each other from different species than to their species-specific paralogues. Whenever high quality genome sequences are available (e.g. human, chimp, dog, rat, and fugu), four bestrophin paralogues can be identified. This strongly argues for a high phylogenetic conservation of the bestrophin subfamilies. Missing paralogues in some species may be due to currently incomplete genomic sequences. One exception may be X. laevis, for which a high quality genome sequence has already been released but still this species reveals only three copies of bestrophins, interestingly all highly similar to bestrophin 2. However, as a tetraploid species, X. laevis appears to 
Table 4: Amino acid changes associated with the divergence of human bestrophins

\begin{tabular}{|c|c|c|c|c|}
\hline Comparison & 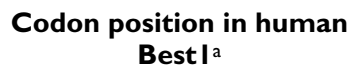 & $\begin{array}{l}\text { Codon position and amino } \\
\text { acid residue }\end{array}$ & $\begin{array}{l}\text { Codon position and amino } \\
\text { acid residue }{ }^{b}\end{array}$ & Protein domainc \\
\hline \multirow[t]{8}{*}{ Bestl vs. Best4 } & & in vertebrate Bestl & in vertebrate Best4 & \\
\hline & 264 & 264: Y, conserved & 279: $P, Q, S, L, E, G$ or $Y$ & Loop 3 \\
\hline & $265^{*}$ & 265: $\mathrm{P}$, conserved & 280: A, T, Q, E or K & Loop 3 \\
\hline & 266 & 266: G, conserved & $28 \mathrm{I}: \mathrm{L}, \mathrm{A}, \mathrm{V}, \mathrm{P}, \mathrm{K}, \mathrm{E}$ or $\mathrm{D}$ & Loop 3 \\
\hline & 267 & 267: H, conserved & 282: G, W, P or $\mathrm{H}$ & Loop 3 \\
\hline & $331^{*}$ & $33 \mathrm{I}: \mathrm{R}, \mathrm{P}, \mathrm{V}, \mathrm{I}, \mathrm{L}$ or $\mathrm{M}$ & 346: $\mathrm{P}$, conserved & C-terminus \\
\hline & 343 & 343: $\mathrm{P}$, conserved & 358: P, A, F or $V$ & C-terminus \\
\hline & 349 & 349: A, conserved & 364: V, I, L, P, Q or A & C-terminus \\
\hline \multirow[t]{15}{*}{ Best 2 vs Best 4} & & in vertebrate Best2 & in vertebrate Best4 & \\
\hline & $45^{*}$ & 45: A, conserved & 45: V, L, M, F or $\mathrm{T}$ & TMD2 \\
\hline & $52^{*}$ & 52: $\mathrm{F}$, conserved & 52: $L, K, R, C$ or $F$ & Loop I \\
\hline & 59 & 59: $\mathrm{K}$, conserved & 59: R, K or $\mathrm{Q}$ & Loop I \\
\hline & $61^{*}$ & 6I: $\mathrm{Y}$, conserved & $6 \mathrm{I}: \mathrm{V}, \mathrm{I}, \mathrm{L}, \mathrm{M}$ or $\mathrm{E}$ & Loop I \\
\hline & 66 & 66: $\mathrm{V}, \mathrm{A}$ or $\mathrm{S}$ & 66: A, conserved & Loop I \\
\hline & 91 & 91: $\mathrm{N}$ or $\mathrm{H}$ & $91: \mathrm{N}$, conserved & Loop 2 \\
\hline & 106 & 106: L or V & 106: L, conserved & Loop 2 \\
\hline & 132 & 132: A, S or C & 132: A, conserved & Loop 2 \\
\hline & 206 & 206: $A, T$ or $S$ & 206: A, conserved & Loop 2 \\
\hline & $207^{*}$ & 207: L, F or $Y$ & 207: L, conserved & Loop 2 \\
\hline & $263^{*}$ & 263: G, conserved & 263: G, E, A or $P$ & Loop 3 \\
\hline & 264 & 264: $Y$, conserved & 279: $P, E, Q, G, S$ or $Y$ & Loop 3 \\
\hline & 331 & $331: \mathrm{V}, \mathrm{M}$ or $\mathrm{P}$ & 346: $\mathrm{P}$, conserved & C-terminus \\
\hline & 349 & 349: A, conserved & 364: $V, P, Q, L$ or $A$ & C-terminus \\
\hline \multirow[t]{7}{*}{ Best 3 vs Best 4} & & in vertebrate Best3 & in vertebrate Best 4 & \\
\hline & $60^{*}$ & 60: R, conserved & 60: $Y, H, R, S$ or $D$ & Loop I \\
\hline & 115 & II5: H, Q, R or L & II5: H, conserved & Loop 2 \\
\hline & $209 *$ & 209: S, T, M or N & 209: L, conserved & Loop 2 \\
\hline & 264 & 264: $Y$, conserved & 279: $P, Q, S, L, E, G$ or $Y$ & Loop 3 \\
\hline & 266 & 266: G, conserved & $28 \mathrm{I}: \mathrm{L}, \mathrm{V}, \mathrm{E}, \mathrm{P}, \mathrm{K}, \mathrm{D}$ or $\mathrm{A}$ & Loop 3 \\
\hline & 331 & $33 \mathrm{I}: \mathrm{K}$ or $\mathrm{R}$ & 346: $\mathrm{P}$, conserved & C-terminus \\
\hline \multirow[t]{6}{*}{ Best 2 vs Best 3} & & in vertebrate Best2 & in vertebrate Best3 & \\
\hline & $165^{*}$ & I65: R, conserved & I65: T, A, P, S or R & Loop 2 \\
\hline & 268 & 268: D, T, S or $N$ & 268: D, conserved & Loop 3 \\
\hline & 333 & 333: $\mathrm{E}$, conserved & 333: $K, R, E, A, N$ or $T$ & C-terminus \\
\hline & $354 *$ & 354: Q, L, D or V & 354: $\mathrm{Y}$, conserved & C-terminus, $\mathrm{Al}$ domain \\
\hline & 365 & 366: $D$, conserved & 365: Q, D, E or H & C-terminus, $\mathrm{Al}$ domain \\
\hline
\end{tabular}

have undergone unique evolutionary changes. Another exception may be the murine bestrophin 4 (gene name: VMD2-L2) which our group has previously shown to represent a functionless pseudogene [33].

In the vertebrate phylogeny, bestrophins 1 and 3 have separated and diverged at a higher rate than bestrophins 2 and 4 . To estimate the selection forces behind this we calculated the substitution rate ratios for the four subgroups. Calculating $\mathrm{dN} / \mathrm{dS}$ ratios across the entire length of the $\mathrm{N}$ - terminal amino acid sequences (aa1-367), we could not, however, find an indication for positive selection. In contrast, the low $\mathrm{dN} / \mathrm{dS}$ values imply that a strong purifying selection may lead to the high sequence conservation observed. Examples such as the chaperonins [24] show that positive selection reaches $\mathrm{dN} / \mathrm{dS}$ values well above 1 for a high selective pressure to fix amino acid substitutions in evolution. Nevertheless, we detected positive selection at several amino acid sites located predominantly in the hydrophilic regions of bestrophin. Testing several models 
only one (M7vsM8) suggested positively selected sites with high posterior probabilities. Another comparison (M0 vs M3), although significant failed to identify positively selected residues with high posterior probability. This points to insufficient information from the alignments or, alternatively, may be explained by the fact that purifying selection in conserved regions could have masked single diversification signals.

Posterior probability analysis for pairwise comparisons of bestrophin paralogues identified significant functional divergence between bestrophin 2 and bestrophin 3 as well as between bestrophin 4 and its remaining paralogues. Generally, the specific amino acid substitutions in the bestrophin paralogues suggest an optimization of protein function, and/or subfunctionalization of the gene duplicates. Eleven out of the 44 positively selected sites were also found to be functionally divergent between bestrophins. The most prominent finding of the posterior probability analysis is that bestrophin 4 diverged significantly during evolution in a number of amino acids from its paralogues. As shown previously [34], bestrophin 4 can be activated by free $\mathrm{Ca}^{2+}$ on the cytoplasmic side although the kinetics of the activation/deactivation is much slower than for typical $\mathrm{Ca}^{2+}$-activated chloride currents [35]. Furthermore, heterologously expressed human bestrophin 4 shows very slow voltage-dependant current relaxations, in contrast to the lack of voltage-dependent current relaxations by human bestrophin 1 and 2. These distinctive properties of bestrophin 4 could be attributable to the additional 15 unique amino acids within the extracellular loop between putative transmembrane domain 5 and 6 . This loop contains three positively charged lysine residues (K266, K269 and K272) within a proline rich region (P267, P273, P277 and P279). It can be speculated that the function of the divergent P279 residue could be to maintain structural or conformational stability of the loop region.

Human bestrophin 1 and 3 have considerably longer amino acid sequences compared to bestrophin 2 and 4 (585 and 668 vs 509 and 473 aa). Human and mouse bestrophin 3, heterologously expressed in HEK-293 cells, produce no chloride currents in a physiological range $[16,36]$, in contrast for example to mouse bestrophin 2 $[13,14]$. Such a functional divergence has been explained to be mediated by an auto-inhibitory (AI) domain composed of seven critical residues ${ }_{356}$ IPSFLGS $_{362}$ present in mouse bestrophin 3 [36]. Interestingly 2 out of 5 amino acid residues implicated in the functional divergence between bestrophin 2 and 3 are located in close proximity to this highly conserved region (Q354 and D366 in bestrophin 2, respectively).

\section{Conclusion}

This study addresses the evolutionary history of the bestrophin protein family. The precise functional properties of the four family members are still unclear but may comprise activities as $\mathrm{Ca}^{2+}$-activated chloride channels or aspects of $\mathrm{Ca}^{2+}$ channel regulation. Functional divergence between the paralogous bestrophins suggests that the bestrophin family members have evolved different functional properties after gene duplication events which have occurred early in the vertebrate lineage. This is most evident for bestrophin 4 where a number of experimental studies provide further support for the in silico data. Our study also demonstrates that amino acids critical for functional divergence are located in regions of the proteins that are likely accessible to soluble ligands, supporting the biochemical and physiological significance of this prediction.

\section{Methods \\ Data collection}

PSI-BLAST and TBLASTN searches with protein sequences of the four human bestrophins were performed in protein databases or unfinished genome sequencing projects at NCBI [37], ENSEMBL [38], the Sanger Institute [39], UCSC Genome Bioinformatics Group [40], and the Joint Genome Institute [41]. Proteins identified by the BLAST search algorithms were considered as potential homologues when amino acid identity was above $25 \%$ over a stretch of $=200$ amino acids. After removal of expressed sequence tags, splice variants and redundant sequences, the initial data set included 173 distinct sequences from 43 species (see Additional file 1).

\section{Sequence alignment and phylogenetic tree reconstruction} Amino acid sequences were aligned with CLUSTAL W (version 1.82) [42] and alignments were refined manually in Genedoc [43]. Incomplete sequences, and highly divergent regions or gaps resulting in uncertain alignments were excluded from the analysis. The final data set includes a total of 53 sequences from 21 vertebrate species (Table 1). A total of $367 \mathrm{~N}$-terminal amino acids were aligned for further studies. Accurate nucleotide alignments were obtained with PAL2NAL [44], a program which constructs multiple codon alignments from matching protein sequences. ProtTest v1.4 [45], implementing the Akaike Information criterion (AIC) was used to estimate the most appropriate model of amino acid substitution for tree building analyses. The best fit model of protein evolution for the bestrophin protein family according to ProtTest corresponds to a JTT $+\mathrm{I}+\mathrm{G}+\mathrm{F}$ model [19].

Tree reconstructions were done by the Neighbor-joining method (NJ) [20] from the protein alignment done in the MEGA v3.1 software package, with the gamma distribu- 
tion model implemented to account for heterogeneity among sites [46], and rooted with the bestrophins from Urohordata. The shape parameter of the gamma distribution $(\alpha)$ was estimated using baseml from the PAMLv4.0 [47] to be $\alpha=0.51$. Support for each phylogenetic group was tested using 1,000 bootstrap pseudoreplicates. Tree topology assessed by maximum parsimony (using Mega v3.1), was substantial similar with the $\mathrm{NJ}$ tree.

\section{Topological structure prediction}

Topological structure prediction was done with the TOPPRED II software [48], which is based on the Kyte and Doolittle algorithm [49]. The average hydrophobicity values of putative transmembrane domains of 20-23 amino acid residues were calculated according the Eisenberg scale [50]. An average hydropathy plot of 53 bestrophinrelated protein sequences was generated by the PEPWINDOWALL program with a window of 19 amino acids [49].

\section{Positive selection assessment}

DNA sequences and related multiple protein sequence alignments were submitted to the PAL2NAL web server [51] which converts a multiple sequence alignment of proteins and the corresponding DNA sequences into a codon alignment. The resulting codon alignments and $\mathrm{NJ}$ tree were used in the program codeml from the PAMLv4.0 software package [47] to calculate the $\mathrm{dN} / \mathrm{dS}$ ( or $\omega$ ) ratio for each site and to test different evolutionary models. The site specific models recommended by Anisimova et al. [26] were tested: Model M0 (one ratio), M1a (nearly neutral), M2a (positive selection), M3 (discrete), M7 (beta) and M8 (beta+ $\omega)$. Model M0 assumed a constant $\omega$-ratio, while in models M1a and M2a $\omega$-ratio is estimated from the date ( 0 $<\omega_{0}<1$ ) while $\omega_{1}=1$ is fixed. M7 and M8 assume a $\beta$-distribution for the $\omega$-value between 0 and 1 . Models $\mathrm{M} 2 \mathrm{a}$, $\mathrm{M} 3$, and M8 allow the occurrence of positively selected sites $(\omega>1)$. Subsequent likelihood rate comparisons of M0 with M3, M1a with $M 2 a$, and M7 with M8, respectively, were performed to test which model fits the data significantly better. Twice the difference in log likelihood between the models is compared with a chi-square distribution with $\mathrm{n}$ degrees of freedom, $\mathrm{n}$ being the difference between the numbers of parameters of the two models.

\section{Functional divergence and detection of amino acids critical for altered functional constraints}

Bestrophin sequence duplication events were tested for type I functional divergence [52] based on the method by $\mathrm{Gu}$ et al [27]. The analysis was carried out with Diverge (version 2.0) [28]. This method is based on maximum likelihood procedures to estimate significant changes in the rate of evolution after the emergence of two paralogous sequences. Type I sites represent amino acid residues conserved in one subfamily but highly variable in another, implying that these residues have been subjected to different functional constraints. A set of 53 protein sequences was included in the study (Table 1, see Additional file 2). Due to gaps and a shorter length of bestrophin 2 and 4 , a total of 15 residues from human bestrophin 4 (codons 264-278) and one (codon 356) from human bestrophin 2 were excluded from the analysis. Consequently, each bestrophin paralogue was restricted to 367 amino acid residues. A new NJ tree was constructed within Diverge with Poisson distance and rerooted. The coefficient of functional divergence $(\theta)$ and the posterior probability for the functional divergence were calculated for each position in the alignment. To detect amino acid residues reflecting functional divergence, bestrophin subfamilies were pair-wise compared to each other. The cut-off value for the posterior probability was determined by consecutively eliminating the highest scoring residues from the alignment until the coefficient of functional divergence dropped to zero.

\section{Authors' contributions}

VMM performed the bioinformatics analyses including all sequence alignments and statistical evaluations. He drafted the manuscript. TL was involved in conceptual discussions for the entire study and made major revisions to the manuscript. RS and KK contributed to the study concept and corrected the manuscript. BHFW participated in conception and design of the study and revised and finalized the manuscript critically. All authors read and approved the final manuscript.

\section{Additional material}

\section{Additional file 1}

List of 173 bestrophin homologues identified in public databases. This table lists the molecular features of all 173 bestrophin homologues identified in public databases.

Click here for file

[http://www.biomedcentral.com/content/supplementary/14712148-8-72-S1.doc]

\section{Additional file 2}

Multiple sequence alignment of vertebrate bestrophins used for calculations of functional divergence. Protein sequences of 53 homologues of vertebrate bestrophins were aligned using the ClustalW algorithm. Shown is the amino acid sequence alignment after removal of gaps and highly divergent regions. Numbering is given according to human bestrophin 1. Sites with predicted functional divergence between bestrophin subfamilies are highlighted by vertical yellow boxes.

Click here for file

[http://www.biomedcentral.com/content/supplementary/14712148-8-72-S2.pdf]

\section{Acknowledgements}

This study was supported by grants from the Deutsche Forschungsgemeinschaft to BHFW (WEI259/13-2 and WEI259/16-2) and to RS (SFB699 Project A7). 


\section{References}

I. Sonnhammer EL, Durbin R: Analysis of protein domain families in Caenorhabditis elegans. Genomics 1997, 46:200-216.

2. Hagen AR, Barabote RD, Saier MH: The bestrophin family of anion channels: identification of prokaryotic homologues. Mol Membr Biol 2005, 22:291-302.

3. Marquardt A, Stohr H, Passmore LA, Kramer F, Rivera A, Weber BH: Mutations in a novel gene, VMD2, encoding a protein of unknown properties cause juvenile-onset vitelliform macular dystrophy (Best's disease). Hum Mol Genet 1998, 7:1517-1525

4. Petrukhin K, Koisti MJ, Bakall B, Li W, Xie G, Marknell T, Sandgren O, Forsman K, Holmgren G, Andreasson S, Vujic M, Bergen AA, GartyDugan V, Figueroa D, Austin CP, Metzker ML, Caskey CT, Wadelius $C$ : Identification of the gene responsible for Best macular dystrophy. Nat Genet 1998, 19:24I-247.

5. The VMD2 database [http://www-huge.uni-regensburg.de/ VMD2 database/]

6. White $\mathrm{K}$, Marquardt A, Weber $\mathrm{BH}$ : VMD2 mutations in vitelliform macular dystrophy (Best disease) and other maculopathies. Hum Mutat 2000, I 5:301-308.

7. Marmorstein AD, Marmorstein LY, Rayborn M, Wang X, Hollyfield JG, Petrukhin K: Bestrophin, the product of the Best vitelliform macular dystrophy gene (VMD2), localizes to the basolateral plasma membrane of the retinal pigment epithelium Proc Natl Acad Sci U S A 2000, 97:I 2758-I 2763

8. Mullins RF, Kuehn MH, Faidley EA, Syed NA, Stone EM: Differentia macular and peripheral expression of bestrophin in human eyes and its implication for best disease. Invest Ophthalmol Vis Sci 2007, 48:3372-3380.

9. Barro SR, Spitzner M, Schreiber R, Kunzelmann K: Bestrophin I enables $\mathrm{Ca2}+$ activated $\mathrm{Cl}$ - conductance in epithelia. I Biol Chem 2006.

10. Pifferi S, Pascarella G, Boccaccio A, Mazzatenta A, Gustincich S, Menini A, Zucchelli S: Bestrophin-2 is a candidate calcium-activated chloride channel involved in olfactory transduction. Proc Nat Acad Sci U S A 2006, 103: I2929-12934.

II. Fischmeister R, Hartzell HC: Volume sensitivity of the bestrophin family of chloride channels. J Physiol 2005 , 562:477-491.

12. Qu Z, Wei RW, Mann W, Hartzell HC: Two bestrophins cloned from Xenopus laevis oocytes express $\mathrm{Ca}(2+)$-activated $\mathrm{Cl}(-)$ currents. J Biol Chem 2003, 278:49563-49572.

13. $\mathrm{Qu} \mathrm{Z}$, Hartzell C: Determinants of anion permeation in the second transmembrane domain of the mouse bestrophin-2 chloride channel. J Gen Physiol 2004, I 24:37|-382.

14. Qu Z, Fischmeister R, Hartzell C: Mouse bestrophin-2 is a bona fide $\mathrm{Cl}(-)$ channel: identification of a residue important in anion binding and conduction. I Gen Physiol 2004, I 23:327-340.

15. Sun $\mathrm{H}$, Tsunenari T, Yau KW, Nathans J: The vitelliform macular dystrophy protein defines a new family of chloride channels. Proc Natl Acad Sci U S A 2002, 99:4008-40I3.

16. Tsunenari T, Sun H, Williams J, Cahill H, Smallwood P, Yau KW, Nathans ]: Structure-function analysis of the bestrophin family of anion channels. I Biol Chem 2003, 278:4 II I 4-4 II 25.

17. Marmorstein LY, Wu J, McLaughlin P, Yocom J, Karl MO, Neussert R, Wimmers S, Stanton JB, Gregg RG, Strauss O, Peachey NS, Mar morstein $A D$ : The light peak of the electroretinogram is dependent on voltage-gated calcium channels and antagonized by bestrophin (best-I). I Gen Physiol 2006, I 27:577-589.

18. Eisen JA: Phylogenomics: improving functional predictions for uncharacterized genes by evolutionary analysis. Genome Res 1998, 8:163-167.

19. Jones DT, Taylor WR, Thornton JM: The rapid generation of mutation data matrices from protein sequences. Comput Appl Biosci 1992, 8:275-282.

20. Saitou N, Nei M: The neighbor-joining method: a new method for reconstructing phylogenetic trees. Mol Biol Evol 1987, 4:406-425.

21. Dehal P, Boore JL: Two rounds of whole genome duplication in the ancestral vertebrate. PLoS Biol 2005, 3:e3 I4.

22. Van de PY: Computational approaches to unveiling ancient genome duplications. Nat Rev Genet 2004, 5:752-763.

23. Milenkovic VM, Rivera $\mathrm{A}$, Horling $\mathrm{F}$, Weber $\mathrm{BH}$ : Insertion and topology of normal and mutant bestrophin-I in the endoplasmic reticulum membrane. J Biol Chem 2007, 282: | 3 | 3- I32 I.
24. Fares MA, Wolfe $\mathrm{KH}$ : Positive selection and subfunctionalization of duplicated CCT chaperonin subunits. Mol Biol Evol 2003, 20:1588-1597.

25. Yang Z: Likelihood ratio tests for detecting positive selection and application to primate lysozyme evolution. Mol Biol Evol 1998, 15:568-573.

26. Anisimova M, Bielawski JP, Yang Z: Accuracy and power of the likelihood ratio test in detecting adaptive molecular evolution. Mol Biol Evol 200I, I 8: I585-I592.

27. Gu X: Statistical methods for testing functional divergence after gene duplication. Mol Biol Evol 1999, 16:1664-1674.

28. Gu X: Maximum-likelihood approach for gene family evolution under functional divergence. Mol Biol Evol 200I, I 8:453-464

29. Gu X, Wang Y, Gu J: Age distribution of human gene families shows significant roles of both large- and small-scale duplications in vertebrate evolution. Nat Genet 2002, 3 I:205-209.

30. Li WH, Gu Z, Wang H, Nekrutenko A: Evolutionary analyses of the human genome. Nature 200I, 409:847-849.

31. Stanton JB, Goldberg AF, Hoppe G, Marmorstein LY, Marmorstein AD: Hydrodynamic properties of porcine bestrophin-I in Triton X-I 00. Biochim Biophys Acta 2006, I 758:24I-247.

32. Qu Z, Chien LT, Cui Y, Hartzell HC: The anion-selective pore of the bestrophins, a family of chloride channels associated with retinal degeneration. J Neurosci 2006, 26:54 I I-54I9.

33. Kramer F, Stohr H, Weber BH: Cloning and characterization of the murine Vmd2 RFP-TM gene family. Cytogenet Genome Res 2004, 105:107-114.

34. Tsunenari T, Nathans J, Yau KW: Ca2+-activated $\mathrm{Cl}$ - current from human bestrophin- 4 in excised membrane patches. Gen Physiol 2006, I 27:749-754.

35. Reisert J, Bauer PJ, Yau KW, Frings S: The Ca-activated Cl channel and its control in rat olfactory receptor neurons. J Gen Physiol 2003, I 22:349-363.

36. Qu ZQ, Yu K, Cui YY, Ying C, Hartzell C: Activation of bestrophin $\mathrm{Cl}$ - channels is regulated by $\mathrm{C}$-terminal domains. I Biol Chem 2007, 282: 17460-17467.

37. The NCBI BLAST database [http://www.ncbi.nlm.nih.gov/ BLAST]

38. The ENSEMBL database [http://www.ensembl.org/index.html]

39. The Sanger Institute Sequencing Projects Blast Search Services [http://www.sanger.ac.uk/DataSearch/blast.shtml]

40. The UCSC Genome browser [http://genome.ucsc.edu/]

4I. The Joint Genome Institute [http://genome.jgi-psf.org/Cioin2/ Cioin2.home.html]

42. Thompson JD, Higgins DG, Gibson TJ: CLUSTAL W: improving the sensitivity of progressive multiple sequence alignment through sequence weighting, position-specific gap penalties and weight matrix choice. Nucleic Acids Res 1994, 22:4673-4680.

43. The National Resource for Biomedical Supercomputing [http://www.psc.edu/biomed/genedoc]

44. Suyama M, Torrents D, Bork P: PAL2NAL: robust conversion of protein sequence alignments into the corresponding codon alignments. Nucleic Acids Res 2006, 34:W609-W6I2.

45. Abascal F, Zardoya R, Posada D: ProtTest: selection of best-fit models of protein evolution. Bioinformatics 2005, 21:2 104-2 I 05.

46. Kumar S, Tamura K, Nei M: MEGA3: Integrated software for Molecular Evolutionary Genetics Analysis and sequence alignment. Brief Bioinform 2004, 5: I50-163.

47. Yang Z: PAML: a program package for phylogenetic analysis by maximum likelihood. Comput Appl Biosci 1997, I 3:555-556.

48. Claros MG, von HG: TopPred II: an improved software for membrane protein structure predictions. Comput Appl Biosci 1994, 10:685-686.

49. Kyte J, Doolittle RF: A simple method for displaying the hydropathic character of a protein. J Mol Biol 1982, I57:105-132.

50. Eisenberg D, Schwarz E, Komaromy M, Wall R: Analysis of membrane and surface protein sequences with the hydrophobic moment plot. J Mol Biol 1984, I 79: I25-|42.

51. The PAL2NAL webserver [http://coot.embl.de/pal2nal]

52. Wang $Y, G u X$ : Functional divergence in the caspase gene family and altered functional constraints: statistical analysis and prediction. Genetics 200I, | 58:|3||-|320. 